Result. A statistically significant association is shown between participants who have had exposure to cannabis and participants who have not had any exposure in their lifetime. The differences across the prioritised brain regions of interest were robust, the association appearing more apparent and statistically significant in the total $(\mathrm{p}=.00)$ and temporal grey matter $(\mathrm{p}=.00)$ regions of the brain. This may suggest that cannabis exposure influences the [18F]DPA-714 VT in the significant regions of interest. However, a negative association is seen with current use, the quantity of use, and the frequency of use.

Conclusion. The initial findings for cannabis exposure show us a positive association with increased TSPO levels, however, limitations must be taken into account. Although we cannot readily establish that elevated TSPO levels in cannabis users can presently act as a risk factor marker for developing psychosis from this particular study, we can utilise this data to continue our research in disclosing a new system to predict the occurrence of psychosis.

\section{The mental health of elite rugby players (a literature review)}

Kirk Musgrave

TEWV NHS Foundation Trust

doi: $10.1192 /$ bjo.2021.731

Aims. Players are Rugby's key asset, what recent research has been conducted into the Mental Health of rugby players/former players?

Method. Initially a Literature Search using HDAS, Ebsco, Researchgate and Googlescholar followed by a Literature Review of relevant articles.

Result. A significantly higher prevalence of anxiety and depressive symptoms in Professional rugby players (compared to the general population) is something that authors agree on. This review considers some of the rugby specific variables not limited to injuries (including concussion), retirement from the sport and finally alcohol abuse.

In 2014, Sullivan looked at the role of potential mediators between concussion and later life depression. Sullivan suggested that the effects of concussion on later life depression may be directly neurological.

Chronic Traumatic Encephalopathy (CTE) is a neurodegeneration which is only definitively diagnosed by post-mortem examination of brain tissue at this time. Today, Chronic Traumatic Encephalopathy is a very controversial subject, for every piece of research which claims to prove CTE, there is another piece of research apparently disproving it.

Alcohol Misuse - Whilst it is well known in general adult psychiatry that alcohol has a significant negative impact on depression and anxiety in the general population, this review summarises findings from research into alcohol misuse in elite rugby players.

Conclusion. In addition to personal variables (which include personality, perfectionism, ability to cope with stress, optimism, pessimism, ability to utilise mental skills, burnout and career satisfaction) there are rugby specific variables which are not limited to injuries, retirement from the sport and finally alcohol abuse.As mentioned in the paragraph on depression and anxiety, numerous recently published authors agree that a significantly higher prevalence of anxiety and depressive symptoms are seen in Professional rugby players (compared to the general population).

As alcohol misuse has already been researched, there would seem to be an opportunity for future research into the extent of illicit drug use by elite rugby players and potentially the effect of illicit drug use on depressive symptoms and anxiety. As mentioned in the paragraph on depression and anxiety, numerous recently published authors agree that a significantly higher prevalence of anxiety and depressive symptoms are seen in Professional rugby players (compared to the general population).

Finally, given the limited recent published literature on suicide in elite rugby players and former elite rugby players, a significant research gap exists in this particular field.

Using electronic clinical records to investigate service use and in-patient care of adults with intellectual disability and/or autism spectrum disorder

Jennifer Mutch ${ }^{1 \star}$, Rory Sheehan ${ }^{2}$, Louise Marston ${ }^{2}$, Nomi Werbeloff ${ }^{2}$, David Osborn ${ }^{2}$ and Angela Hassiotis ${ }^{2}$

${ }^{1}$ NHS Lothian Learning Disability Service and ${ }^{2}$ University College London

${ }^{\star}$ Corresponding author.

doi: $10.1192 /$ bjo.2021.732

Aims. To describe characteristics of adults with intellectual disability (ID) and/or autism spectrum disorder (ASD) accessing care in one mental health Trust.

To explore factors associated with in-patient admission/risk of re-admission within 12 months of discharge.

Background. There is concern that adults with intellectual disability and those with autism spectrum disorder are frequently admitted to mental health hospitals. The evidence from NHS datasets suggests that this remains a significant issue and is associated with personal, social and economic costs.

Method. Adults ( $\geq 18$ years) with ICD-10 diagnosis of "mental retardation" and/or autism who had accessed care in the Camden and Islington Foundation Trust were identified using the Clinical Record Interactive Search (CRIS). The identification process was validated through cross checking of free text in the electronic clinical notes. We compared demographic and clinical characteristics and service use, including length of admission, of 315 individuals with ASD and 339 with ID (with or without ASD). Logistic regression was used to explore factors associated with in-patient admission and re-admission within 12 months of discharge.

Result. A greater proportion of adults with ID (with or without ASD) had a diagnosis of psychosis, substance misuse, or dementia whereas diagnosis of anxiety disorder was greater in those with ASD. Antipsychotics and other psychotropics were twice as likely to be prescribed for the ID \pm group. Admission to psychiatric in-patient care was greater in those with ID \pm ASD (adjusted OR 4.00, 95\% confidence interval (CI) 2.41-6.63), men (aOR $2.28,95 \% \mathrm{CI} 1.39-3.75$ ), younger adults (aOR 0.98, 95\%CI 0.97-1.00), and in those with a diagnosis of schizophrenia spectrum disorder (aOR 5.08, 95\%CI 3.00-8.61), affective disorder (aOR 2.23, 95\%CI 1.29-3.83), personality disorder (aOR 1.94, 95\%CI 1.02-3.68), and record of previous inpatient admission (aOR 2.18, 95\%CI 1.17-4.05). Having ASD alone was associated with a greater risk of re-admission within one year of discharge, although this difference was not statistically significant (aOR $0.70,95 \%$ CI 0.32-1.52). Comorbid diagnoses of affective disorder or personality disorder were the only significant associations with re-admission (aOR 3.11, 95\%CI 1.34-7.23 and aOR 8.28, 95\%CI 2.85-24.04, respectively).

Conclusion. These findings provide the first longitudinal investigation into the acute care pathway for adults with ID and/or ASD in the NHS. Replication in other trusts is now needed to inform "at risk of admission" registers and guide targeted interventions to prevent admission. 